\title{
Polyphosphoester nanoparticles as biodegradable platform for delivery of multiple drugs and siRNA
}

This article was published in the following Dove Press journal:

Drug Design, Development and Therapy

22 February 2017

Number of times this article has been viewed

\author{
Hadeel Elzeny',* \\ Fuwu Zhang ${ }^{2, *}$ \\ Esraa $\mathrm{N} \mathrm{Ali}^{1}$ \\ Heba A Fathi' \\ Shiyi Zhang ${ }^{3}$ \\ Richen $\mathrm{Li}^{2}$ \\ Mohamed A El-Mokhtar ${ }^{4}$ \\ Mostafa A Hamad ${ }^{5}$ \\ Karen LWooley ${ }^{2,6}$ \\ Mahmoud Elsabahy ${ }^{1,6-8}$ \\ 'Assiut International Center of \\ Nanomedicine, Al-Rajhy Liver \\ Hospital, Assiut University, Assiut, \\ Egypt; ${ }^{2}$ Departments of Chemistry, \\ Chemical Engineering and Materials \\ Science and Engineering, Texas \\ A\&M University, College Station, \\ TX, USA; 3 School of Biomedical \\ Engineering, Shanghai Jiao Tong \\ University, Shanghai, People's \\ Republic of China; ${ }^{4}$ Department \\ of Microbiology and Immunology, \\ Faculty of Medicine, ${ }^{5}$ Department of \\ Surgery, Faculty of Medicine, Assiut \\ University, Assiut, Egypt; ' ${ }^{6}$ aboratory \\ for Synthetic-Biologic Interactions, \\ Department of Chemistry, Texas \\ A\&M University, College Station, TX, \\ USA; ${ }^{7}$ Department of Pharmaceutics, \\ Faculty of Pharmacy, Assiut University, \\ Assiut, ${ }^{8}$ Misr University for Science \\ and Technology, 6th of October \\ City, Egypt \\ *These authors contributed equally \\ to this work
}

Correspondence: Mahmoud Elsabahy; Karen L Wooley

Department of Pharmaceutics,

Faculty of Pharmacy, Assiut University,

Assiut 71515, Egypt

Tel +201000607466

Fax +20 8820807 II

Email mahmoud.elsabahy@chem.tamu. edu; wooley@chem.tamu.edu

\begin{abstract}
Delivery of multiple therapeutics and/or diagnostic agents to diseased tissues is challenging and necessitates the development of multifunctional platforms. Among the various strategies for design of multifunctional nanocarriers, biodegradable polyphosphoester (PPE) polymers have been recently synthesized via a rapid and simple synthetic strategy. In addition, the chemical structure of the polymer could be tuned to form nanoparticles with varying surface chemistries and charges, which have shown exceptional safety and biocompatibility as compared to several commercial agents. The purpose of this study was to exploit a mixture of PPE nanoparticles of cationic and neutral surface charges for multiple delivery of anticancer drugs (ie, sorafenib and paclitaxel) and nucleic acids (ie, siRNA). Cationic PPE polymers could efficiently complex siRNA, and the stability of the nanoparticles could be maintained in physiological solutions and upon freeze-drying and were able to deliver siRNA in vivo when injected intravenously in mice. Commercially available cationic polyethylenimine polymer had $\mathrm{LD}_{50}$ of ca. $61.7 \mathrm{mg} / \mathrm{kg}$ in mice, whereas no animal died after injection of the cationic PPE polymer at a dose of $>130 \mathrm{mg} / \mathrm{kg}$. Neutral PPE nanoparticles were able to encapsulate two hydrophobic drugs, namely, sorafenib and paclitaxel, which are commonly used for the treatment of hepatocellular carcinoma. Mixing the neutral and cationic PPE nanoparticles did not result in any precipitation, and the size characteristics of both types of nanoparticles were maintained. Hence, PPE polymers might have potential for the delivery of multiple drugs and diagnostic agents to diseased tissues via simple synthesis of the individual polymers and assembly into nanoparticles that can host several drugs while being mixed in the same administration set, which is of importance for industrial and clinical development.
\end{abstract}

Keywords: biodegradable nanoparticles, polyphosphoester, chitosan, polyethylenimine, siRNA, sorafenib, paclitaxel

\section{Introduction}

Delivery of multiple therapeutics and/or diagnostic agents to diseased tissues is challenging and necessitates the development of multifunctional platforms. ${ }^{1-3}$ Combinational therapy and/or diagnosis are useful in reducing the frequency of administration and improving patient compliance. In addition, for the treatment of some diseases, such as cancer, delivery of multiple drugs allows targeting several pathways of the disease and might overcome resistance to chemotherapy. ${ }^{4-8}$

There are several strategies for codelivery of therapeutic and diagnostic agents. ${ }^{4-8}$ We have recently designed multifunctional hierarchically assembled theranostic nanostructures that undergo radiolabeling and can be loaded with anticancer drug (paclitaxel) and short-interfering RNA (siRNA) and also could be labeled with fluorescent probes. ${ }^{9}$ Design of these nanostructures depended on complexation between cationic nondegradable spherical nanoparticles and siRNA, followed by assembly of 
these spheres on paclitaxel-loaded cylindrical nondegradable anionic nanoparticles. Despite the therapeutic efficiency of multifunctional nanocarriers, developed by our group and others, ${ }^{4-8}$ the synthetic strategies and procedures involved in the construction of these nanocarriers were sophisticated and required tuning the structure of a single type of nanoparticles to accommodate $>1 \mathrm{drug}$ and/or diagnostic probe. Moreover, loading of drugs of varying solubilities and molecular weights (eg, hydrophobic anticancer drugs versus hydrophilic peptides and nucleic acids) could further complicate the procedures involved in the preparation of the drug-loaded nanoparticles and may destabilize or degrade some drugs.

The aim of this study was to test the possibility of loading paclitaxel, sorafenib and siRNA into biodegradable nanoparticles. Paclitaxel is hydrophobic anticancer drug that is commonly used for the treatment of a variety of cancers. Because of its low solubility, it is formulated in high concentration in Cremophor $^{\circledR}$ EL $\left(\right.$ Taxol $\left.^{\circledR}\right)$, which induces hypersensitivity reactions. ${ }^{10}$ Sorafenib is the only US FDAapproved molecularly targeted drug for systemic treatment of advanced hepatocellular carcinoma (HCC) and can sensitize HCC cells to paclitaxel. ${ }^{11-14}$ However, sorafenib also suffers from low aqueous solubility and poor bioavailability. ${ }^{15,16}$ Sorafenib and paclitaxel together with siRNA targeted against specific genes (eg, integrin and vascular endothelial growth factor) ${ }^{17,18}$ can be effective in the management and diagnosis of HCC. Therapeutic uses of siRNA are limited by its low stability, rapid enzymatic degradation and low intracellular bioavailability. ${ }^{19}$

Nanomaterials provide promising tools for the efficient treatment and diagnosis of HCC. ${ }^{20}$ Nanoparticles can afford greater solubility and stability of the hydrophobic anticancer drugs and nucleic acids and allow greater accumulation in the tumor areas. Biodegradable polyphosphoester (PPE) polymers have been recently synthesized via a rapid and simple synthetic strategy. ${ }^{21}$ In addition, the chemical structure of the polymer could be tuned to form nanoparticles with varying surface chemistries and charges, which have shown exceptional safety and biocompatibility as compared to several commercial agents. ${ }^{22}$ In this study, individual nanoparticles loaded with chemotherapeutics (in the neutral nanoparticles) and siRNA (in the cationic nanoparticles) were prepared. Cationic PPE polymers could efficiently interact with polyanionic siRNA via electrostatic complexation, whereas neutral PPE polymers could form nanoparticles that accommodate anticancer drugs in their hydrophobic domains. The purpose of this study was to evaluate the use of mixture of PPE nanoparticles of cationic and neutral surface charges for the multiple delivery of anticancer drugs (ie, sorafenib and paclitaxel) and nucleic acids (ie, siRNA) for potential treatment of HCC.

In this study, PPE nanoparticles loaded with either siRNA or paclitaxel/sorafenib were characterized. Toxicity and efficiency of the PPE nanoparticles were compared with the most commonly used cationic degradable (ie, chitosan) and nondegradable (ie, polyethylenimine [PEI]) polymers for the delivery of siRNA. The effect of mixing the neutral and cationic PPE nanoparticles on size characteristics of the individual nanoparticles was also investigated. Wang et al have explored the application of partially degradable triblock copolymer poly(ethylene glycol)- $b$-poly( $\varepsilon$-caprolactone)$b$-poly(2-aminoethyl ethylene phosphate) for siRNA and paclitaxel delivery. ${ }^{7,23,24}$ In this report, functional PPE polymer with fully degradable backbone has been developed to conveniently construct nanoparticles with varying surface charges, for siRNA and chemotherapeutic delivery. The use of fully degradable PPE nanoparticles for the delivery of siRNA and sorafenib, either as individual drugs or combined, has not been reported previously.

\section{Materials and methods Materials}

The hydrophobic functional block copolymer, poly(2ethylbutoxy phospholane)-block-poly(2-butynyl phospholane) (PEBP- $b$-PBYP, $M_{\mathrm{n}}^{\mathrm{NMR}}=19,300$, PDI $\left.=1.25\right)$, and the amphiphilic block terpolymer, poly(2-ethylbutoxy phospholane)-block-poly(2-butynyl phospholane)-graftpoly(ethylene glycol) (PEBP- $b$-PBYP- $g$-PEG, $M_{\mathrm{n}}^{\mathrm{NMR}}=27,300$, PDI $=1.24$ ), were synthesized as reported previously. ${ }^{21,25}$ Cysteamine hydrochloride and 2,2-dimethoxy-2phenylacetophenone (DMPA) were used as received from Sigma-Aldrich (St Louis, MO, USA). The Spectra/Por dialysis membranes (MWCO 12-14 kDa) were purchased from Spectrum Laboratories, Inc. (Rancho Dominguez, CA, USA). Nanopure water $(18 \mathrm{M} \Omega \cdot \mathrm{cm})$ was acquired by means of a Milli-Q water filtration system, Millipore Corp. (Bedford, MA, USA). Low molecular weight chitosan (50,000-190,000 Da), cyanine 5-labeled siRNA and PEI (branched PEI, average molecular weight of 60,000) were purchased from Sigma-Aldrich. Ultra-pure water (free from RNAse and DNAse) was purchased from Biochrom GmbH (Berlin, Germany). Tris buffer was purchased from Fisher Scientific (Waltham, MA, USA). Ethidium bromide and phosphate-buffered saline (PBS) were purchased from MP Biomedicals (Santa Ana, CA, USA). Tris-borate-EDTA 
(TBE) buffer and agarose (molecular biology grade) were purchased from Fischer Scientific. Dulbecco's Modified Eagle's Medium (DMEM) was purchased from Lonza (Basel, Switzerland). Fetal bovine serum (FBS) was purchased from Biowest (Riverside, MO, USA). Cell titer $96^{\circledR}$ Non-Rad cell proliferation assay (MTT) was purchased from Promega (Madison, WI, USA).

\section{Instrumentation}

${ }^{1} \mathrm{H}$ NMR and ${ }^{13} \mathrm{C}$ NMR spectra were recorded on a Varian Inova 300 or Mercury 300 spectrometer interfaced to a UNIX computer using VnmrJ software. Chemical shifts were referenced to the solvent residual signals. The DMF size exclusion chromatography was conducted on a Waters Chromatography, Inc. (Milford, MA, USA) system equipped with an isocratic pump model 1515, a differential refractometer model 2414 and a three-column set of Styragel HR 4 $5 \mu \mathrm{m}$ DMF $(300 \times 7.5 \mathrm{~mm})$, Styragel HR 4E $5 \mu \mathrm{m}$ DMF $(300 \times 7.5 \mathrm{~mm})$ and Styragel HR 2.5 $\mathrm{mm}$ DMF $(300 \times 7.5 \mathrm{~mm})$. The system was equilibrated at $70^{\circ} \mathrm{C}$ in prefiltered DMF containing $0.05 \mathrm{M} \mathrm{LiBr}$, which served as polymer solvent and eluent (flow rate set to $1.00 \mathrm{~mL} / \mathrm{min}$ ). Polymer solutions were prepared at a concentration of ca. $3 \mathrm{mg} / \mathrm{mL}$, and an injection volume of $200 \mu \mathrm{L}$ was used. Data collection and analysis were performed with Empower 2 v. 6.10.01.00 software (Waters Chromatography, Inc.). The system was calibrated with polystyrene standards (Polymer Laboratories, Amherst, MA, USA) ranging from 615 to $442,800 \mathrm{Da}$.

Glass transition temperatures $\left(\mathrm{T}_{\mathrm{g}}\right)$ were measured by differential scanning calorimetry (DSC) on a Mettler-Toledo DSC $822^{\circledR}$ (Mettler-Toledo, Inc., Columbus, OH, USA), with a heating rate of $10^{\circ} \mathrm{C} / \mathrm{min}$. Measurements were analyzed using Mettler-Toledo Star ${ }^{\mathrm{e}}$ v. 7.01 software. The $\mathrm{T}_{\mathrm{g}}$ was taken as the midpoint of the inflection tangent, upon the third heating scan. Thermogravimetric analysis (TGA) was performed under $\mathrm{N}_{2}$ atmosphere using a Mettler-Toledo model TGA/SDTA $851^{\mathrm{e}}$, with a heating rate of $10^{\circ} \mathrm{C} / \mathrm{min}$. Measurements were analyzed by using Mettler-Toledo Star ${ }^{\mathrm{e}}$ v. 7.01 software.

\section{General procedure of thiol-yne reactions of $\mathrm{PEBP}_{50}-b-\mathrm{PBYP}_{50}$ with cysteamine hydrochloride}

A solution of PEBP $_{50}-b$-PBYP ${ }_{50}(0.70 \mathrm{~g}, 1.8 \mathrm{mmol}$ alkynes $)$, cysteamine hydrochloride (2.9 g, $25 \mathrm{mmol})$, and DMPA (70 mg, $0.27 \mathrm{mmol}$ ) in $20 \mathrm{~mL}$ of DMF was bubbled with nitrogen for $10 \mathrm{~min}$ and then irradiated under UV irradiation $(365 \mathrm{~nm})$ for $2 \mathrm{~h}$. The reaction mixtures were then transferred to dialysis tubing (MWCO 12-14 kDa) and dialyzed against nanopure water with hydrochloride $(\mathrm{pH} 3.0)$ in the cold room $\left(4^{\circ} \mathrm{C}-8^{\circ} \mathrm{C}\right)$ for $36 \mathrm{~h}$ to remove excess cysteamine hydrochloride and other small molecules. The purified micelles were lyophilized to yield sticky liquid (0.90 g, 81\% yield). ${ }^{1} \mathrm{H}$ NMR (d $\left.-\mathrm{DMSO}, \mathrm{ppm}\right): \delta 0.84(\mathrm{t}, \mathrm{J}=7.5 \mathrm{~Hz}$, $\left.\mathrm{POCH}_{2} \mathrm{CH}\left(\mathrm{CH}_{2} \mathrm{CH}_{3}\right)_{2}\right), 1.30\left(\mathrm{~m}, \mathrm{POCH}_{2} \mathrm{CH}\left(\mathrm{CH}_{2} \mathrm{CH}_{3}\right)_{2}\right)$, $1.46\left(\mathrm{~m}, \mathrm{POCH}_{2} \mathrm{CH}\left(\mathrm{CH}_{2} \mathrm{CH}_{3}\right)\right), 1.60-1.87,2.15-2.34$ (b, $\mathrm{POCH}_{2} \mathrm{CH}_{2}$ ), 2.76-3.15 (br, $\mathrm{CH}_{2} \mathrm{SCH}_{2} \mathrm{CHSCH}_{2}, \mathrm{CH}_{2} \mathrm{NH}_{3}$ ), 3.85-4.45 (br, $\mathrm{POCH}_{2} \mathrm{CH}, \mathrm{POCH}_{2} \mathrm{CH}_{2}, \mathrm{POCH}_{2} \mathrm{CH}_{2} \mathrm{OP}$ ), 5.03 (d, $\left.\mathrm{OCH}_{2} \mathrm{Ar}\right), 7.30-7.56$ (m, Ar- $\left.H\right), 8.10-8.55$ (br, $\left.\mathrm{SCH}_{2} \mathrm{CH}_{2} \mathrm{NH}_{3} \mathrm{Cl}\right) \cdot{ }^{13} \mathrm{C} \mathrm{NMR}\left(\mathrm{d}_{6}-\mathrm{DMSO}, \mathrm{ppm}\right)$ : $\delta 10.63,22.18,26.79,28.59,33.30,36.91,40.85,41.33$, $43.44,66.08,65.30,66.10,66.49,68.87,126.63,128.49$, $128.73,129.41,133.21,136.63 .{ }^{31} \mathrm{P}$ NMR (d $\left.-\mathrm{DMSO}, \mathrm{ppm}\right)$ : $\delta-0.98,-1.28$. DSC: $\mathrm{T}_{\mathrm{g}}=-54^{\circ} \mathrm{C}$. $\mathrm{TGA}$ in $\mathrm{N}_{2}: 170^{\circ} \mathrm{C}-265^{\circ} \mathrm{C}$, $41 \%$ mass loss $; 265^{\circ} \mathrm{C}-340^{\circ} \mathrm{C}, 16 \%$ mass loss; $340^{\circ} \mathrm{C}-500^{\circ} \mathrm{C}$, $11 \%$ mass loss, $32 \%$ mass remaining above $500^{\circ} \mathrm{C}$. IR $\left(\mathrm{cm}^{-1}\right)$ : 3,700-3,250, 3,250-2,350, 1,613, 1,510, 1,463, 1,384, 1,251, $1,018,968,803$.

\section{Preparation of PPE nanoparticles loaded with chemotherapeutics}

Sorafenib-loaded polyphosphoester (SOR-PPE) nanoparticles

Sorafenib and PEBP- $b$-PBYP- $g$-PEG PPE terpolymer were dissolved in ethanol at 1:10 drug to polymer weight ratio. Then, the solution was sonicated for $5 \mathrm{~min}$, followed by the removal of ethanol under vacuum and resuspension of the formed film in distilled water, followed by 5 min sonication to allow the formation of well-dispersed nanoparticles.

\section{Paclitaxel-loaded polyphosphoester (PTX-PPE) nanoparticles}

Paclitaxel and PEBP- $b$-PBYP- $g$-PEG PPE terpolymer were dissolved in ethanol at 1:10 drug to polymer weight ratio. Then, nanoparticles were prepared as described for the sorafenib-loaded nanoparticles.

\section{Paclitaxel- and sorafenib-loaded polyphosphoester (PTX-SOR-PPE) nanoparticles}

Paclitaxel and sorafenib at a weight ratio of 1:1 were dissolved in ethanol. Then, PEBP- $b$-PBYP- $g$-PEG PPE terpolymer was dissolved in ethanol and mixed with the drug solution at a weight ratio of total drug to polymer of $1: 10$. Then, nanoparticles were prepared as described for the sorafenib- and paclitaxel-loaded nanoparticles. 


\section{Measurement of the entrapment efficiency (EE)}

EE of drugs in the nanoparticles was measured using a reversed-phase high performance liquid chromatography (RP-HPLC) method. The method was validated for linearity, accuracy and precision. Paclitaxel in ethanol was separated onto RP-HPLC using a $\mathrm{C}_{18}$ column and a mobile phase consisting of acetonitrile/water $(50: 50, \mathrm{v} / \mathrm{v})$ at a flow rate of $1.0 \mathrm{~mL} / \mathrm{min}$. The injection volume was $20 \mu \mathrm{L}$, and eluent was analyzed with a UV detector set at a wavelength of $227 \mathrm{~nm}$. Sorafenib in ethanol was separated onto RPHPLC using a $\mathrm{C}_{18}$ column and a mobile phase consisting of acetonitrile:methanol:1\% acetic acid at a ratio of 35:38:27 at a flow rate of $1.0 \mathrm{~mL} / \mathrm{min}$. The injection volume was $20 \mu \mathrm{L}$, and eluent was analyzed with a UV detector set at a wavelength of $254 \mathrm{~nm}$. After dissociation of nanoparticles by the addition of organic solvent, the drug content in the nanoparticles was determined by RP-HPLC. EE (\%) was calculated according to the following equation:

Encapsulation efficiency (\%)

$=\frac{\text { Amount of drug encapsulated into nanoparticles }}{\text { Amount of drug initially added }} \times 100$

\section{Preparation of siRNA-loaded nanoparticles}

Stock solutions of the cationic block copolymer (PEBP- $b$ PBYP-C) PPE copolymer and siRNA were prepared in Tris buffer $(10 \mathrm{mM}, \mathrm{pH} 7.4,1 \mathrm{mg} / \mathrm{mL})$. About $1.5 \mu \mathrm{L}$ siRNA was mixed with different volumes of the cationic PPE polymer solutions to prepare nanoparticles at different nitrogen/phosphate (N/P) ratios from 0 to 40 . The mixture was vortexed for $1 \mathrm{~min}$ and incubated at room temperature before characterizations. Chitosan/tripolyphosphate (Tpp)-based nanoparticles were prepared by ionic gelation method, and Tpp was incorporated as a crosslinker, at different N/P ratios from 0 to 50 , as described previously. ${ }^{26}$ The PEI-based nanoparticles were prepared as described previously at N/P ratios from 0 to $-50 .{ }^{26}$

\section{Gel retardation assay}

Gel retardation assay was used to evaluate the encapsulation efficiency of siRNA in different types of nanoparticles, which was performed by using gel electrophoresis (Elettrofor, Rovigo RO, Italy), as described previously. ${ }^{26}$ Briefly, nanoparticles prepared at different N/P ratios were run on $1 \%(\mathrm{w} / \mathrm{v})$ agarose gel and electrophoresed using TBE as a running buffer for $1 \mathrm{~h}$ at $100 \mathrm{~V}$. Ethidium bromide was added to the gel as a staining agent, and bands were detected by UV camera that measures the absorbance from 300 to $360 \mathrm{~nm}$ (Biometra, Göttingen, Germany). The experiment was performed in triplicates.

\section{Size and zeta potential measurements}

The mean hydrodynamic diameters and size distribution (polydispersity index [PDI]) of the nanoparticles were measured using a Zetasizer Nano ZS instrument (Malvern Instruments, Worcestershire, UK) equipped with a backscattered light detector operating at $173^{\circ}$ as described previously. The zeta potential values were determined by laser Doppler anemometry using Malvern Zetasizer Nanoseries ZS. All measurements were performed in triplicates. The effect of different concentrations of $\mathrm{NaCl}(0.1-2 \mathrm{M})$ and dextrose $(0.5 \%-10 \%, \mathrm{w} / \mathrm{v})$ solutions on the mean hydrodynamic diameters and size distributions of PPE-, chitosan/Tpp- and PEIbased nanoparticles was assessed, as described previously. ${ }^{26}$ All measurements were performed in triplicates.

Morphology of the nanoparticles was studied by transmission electron microscopy (TEM). TEM images were collected on a JEOL 1200EX operating at $100 \mathrm{kV}$, and micrographs were recorded at calibrated magnifications using an SIA-15C CCD camera. Aqueous solutions of the samples $(4 \mu \mathrm{L})$ were deposited onto carbon-coated copper grids, and after $1 \mathrm{~min}$, the excess of the solution was quickly wicked away by a piece of filter paper. A drop of $1 \mathrm{wt} \%$ uranyl acetate was then added and allowed to stand for $30 \mathrm{~s}$ before excess stain was wicked away. The grids were allowed to dry in air overnight.

\section{Freeze-drying}

The ability of nanoparticles to maintain their characteristics (size and size distribution) upon freeze-drying was evaluated in the presence or absence of cryoprotectants, such as mannitol and sucrose. Water or solutions of different cryoprotectants $(1 \mathrm{~mL})$ were added to $2 \mathrm{~mL}$ of the prepared nanoparticle solutions. The nanoparticles were then frozen at $-80^{\circ} \mathrm{C}$ overnight and lyophilized (Alpha 2.4 LD plus Freeze-dryer, Martin Christ GmbH, Osterode, Germany). The operating conditions were set up at a temperature of $-60^{\circ} \mathrm{C}$ and pressure of $0.011 \mathrm{mbar}$. The mean hydrodynamic diameters and size distributions of the nanoparticles were measured before and after freeze-drying.

\section{Hemolysis test}

Human blood samples were collected from the Assiut University Hospital into EDTA-containing tubes and were centrifuged at 3,000 rpm for $5 \mathrm{~min}$. The pellets were washed three times in isotonic saline $(0.9 \%, \mathrm{w} / \mathrm{v}$ sodium chloride) by centrifugation at 3,000 rpm for $5 \mathrm{~min}$ and were 
resuspended in saline. Suspensions of red blood cells (RBCs) were freshly prepared and used within $24 \mathrm{~h}$ after collection. Ten microliters of different formulations (PTX-SOR-PPE nanoparticles, Taxol, saline and distilled water) were added to erythrocytes $(500 \mu \mathrm{L})$ and then samples were incubated in a water bath shaker at $37^{\circ} \mathrm{C}$ for $2 \mathrm{~h}$ at $300 \mathrm{rpm}$. Then, samples were centrifuged at 5,000 rpm for $10 \mathrm{~min}$ and supernatants were analyzed at $570 \mathrm{~nm}$ for hemoglobin content. Complete hemolysis was achieved using water yielding the $100 \%$ hemolysis control value, whereas saline was used to represent the $0 \%$ hemolysis control. The experiments were repeated in triplicates.

\section{In vitro cytotoxicity assay}

The cytotoxicities of paclitaxel-, sorafenib- and paclitaxel/ sorafenib-loaded PPE nanoparticles were determined on HCC cell line (Huh-7) by the colorimetric MTT assay according to the instructions of the manufacturer's protocol for quantifying cell viability. Huh-7 cells were kindly provided by the Cell Culture Department of VACSERA (Cairo, Egypt). Briefly, the cells $\left(1 \times 10^{5}\right)$ were cultured on 96-well plate in Dulbecco's Modified Eagle's Medium (5\% FBS) supplemented with $4.5 \mathrm{~g} / \mathrm{L}$ glucose, L-glutamine, penicillin and streptomycin. The cells were incubated overnight, followed by the addition of $20 \mu \mathrm{L}$ of serial dilutions of the different nanoparticles. The highest tested concentrations of paclitaxel and sorafenib were $50 \mu \mathrm{M}$. The total volume in each well was $100 \mu \mathrm{L}$. The plates were then incubated at $37^{\circ} \mathrm{C}$ for $24 \mathrm{~h}$ in a humidified atmosphere $\left(5 \% \mathrm{CO}_{2}\right)$. After incubation, $15 \mu \mathrm{L}$ of the MTT (3-(4, 5-dimethyl-thiazol-2-yl)-2, 5-diphenyltetrazolium bromide, $5 \mathrm{mg} / \mathrm{mL}$ ) in sterile-filtered PBS was added to each well to allow for the formation of formazan crystals. After $4 \mathrm{~h}, 100 \mu \mathrm{L}$ of DMSO (solubilization solution) was added to each well to dissolve the formed formazan crystals. Finally, the absorbance at $570 \mathrm{~nm}$ was recorded using a microplate reader after $24 \mathrm{~h}$. The cell viability was calculated as a percentage of viability of the untreated cells as described previously. ${ }^{26}$

\section{Evaluation of the in vivo toxicity and $\mathrm{LD}_{50}$} Experimental albino mice were purchased from the Assiut University Faculty of Medicine Animal Housing Facility. Mice were kept in cages and allowed food and water ad libitum. Toxicity of the PPE, chitosan/Tpp and PEI polymers was assessed after intraperitoneal injection of $200 \mu \mathrm{L}$ of increasing concentrations of the polymers from 0.5 to $4 \mathrm{mg} / \mathrm{mL}$ into mice $(30 \mathrm{~g})$. All preparation processes were carried out in sterile area, and the formulations were prepared in sterile saline. The end point of the experiment was $24 \mathrm{~h}$. The analysis of data and determination of the $\mathrm{LD}_{50}$ were performed by Miller and Tainter method. ${ }^{27-29}$ Experiments were performed at least in triplicates.

Ethical and legal approvals were obtained before the commencement of the study, and all the animal experiments were approved by the Institutional Animal Ethical Committee of the Faculty of Pharmacy, Assiut University, and it adheres to the Guide for the Care and Use of Laboratory Animals, 8th Edition, National Academies Press, Washington, DC, USA. Experiments utilizing blood were approved by the Ethical Committee of the Assiut University, Faculty of Medicine, and written consents were also obtained from the blood donors.

\section{Evaluation of the in vivo biodistribution of siRNA}

Albino mice were purchased and experimental procedures were carried out as described in the previous section. PPE, chitosan/Tpp and PEI polymers were complexed with cyanine 5-fluorescently labeled siRNA to form the nanoparticles. Then, naked siRNA and siRNA-loaded nanoparticles were injected intravenously. Each formulation contained $15 \mu \mathrm{L}$ of fluorescent siRNA at a dose of $0.5 \mathrm{mg}$ siRNA $/ \mathrm{kg}$. ${ }^{30,31}$ Because of the photosensitivity of the fluorescent siRNA, the experiments were performed under dark conditions. The mice were euthanized after $2 \mathrm{~h}$ of the injection. Specimens from the liver, kidney, spleen, lungs and heart of control and treated mice were harvested and fixed immediately, and processed for examination under light and fluorescence microscopy, for histologic examination and in vivo distribution evaluation of siRNA, as described previously. ${ }^{26}$

\section{Results and discussion Synthesis of cationic and neutral PPE polymers}

There has been an increasing interest to develop biodegradable PPE-based nanomaterials as biomedical devices for drug and gene delivery applications. ${ }^{32-35}$ Recently, we have reported on the facile synthesis of a hydrophobic-functional diblock PPE PEBP- $b$-PBYP by sequential ring-opening polymerizations, ${ }^{21}$ a well-established approach to synthesize degradable polymers, such as polyesters, ${ }^{35,36}$ polypeptides $^{37,38}$ and PPEs. ${ }^{39,40}$ This block copolymer could be easily transformed into amphiphic block terpolymer PEBP- $b$-PBYP- $g$ PEG by PEGylation via copper(I)-catalyzed azide-alkyne cycloaddition (CuAAC) in $N, N$-dimethylformamide (Scheme 1). ${ }^{25}$ The diblock copolymer PEBP- $b$-PBYP could 

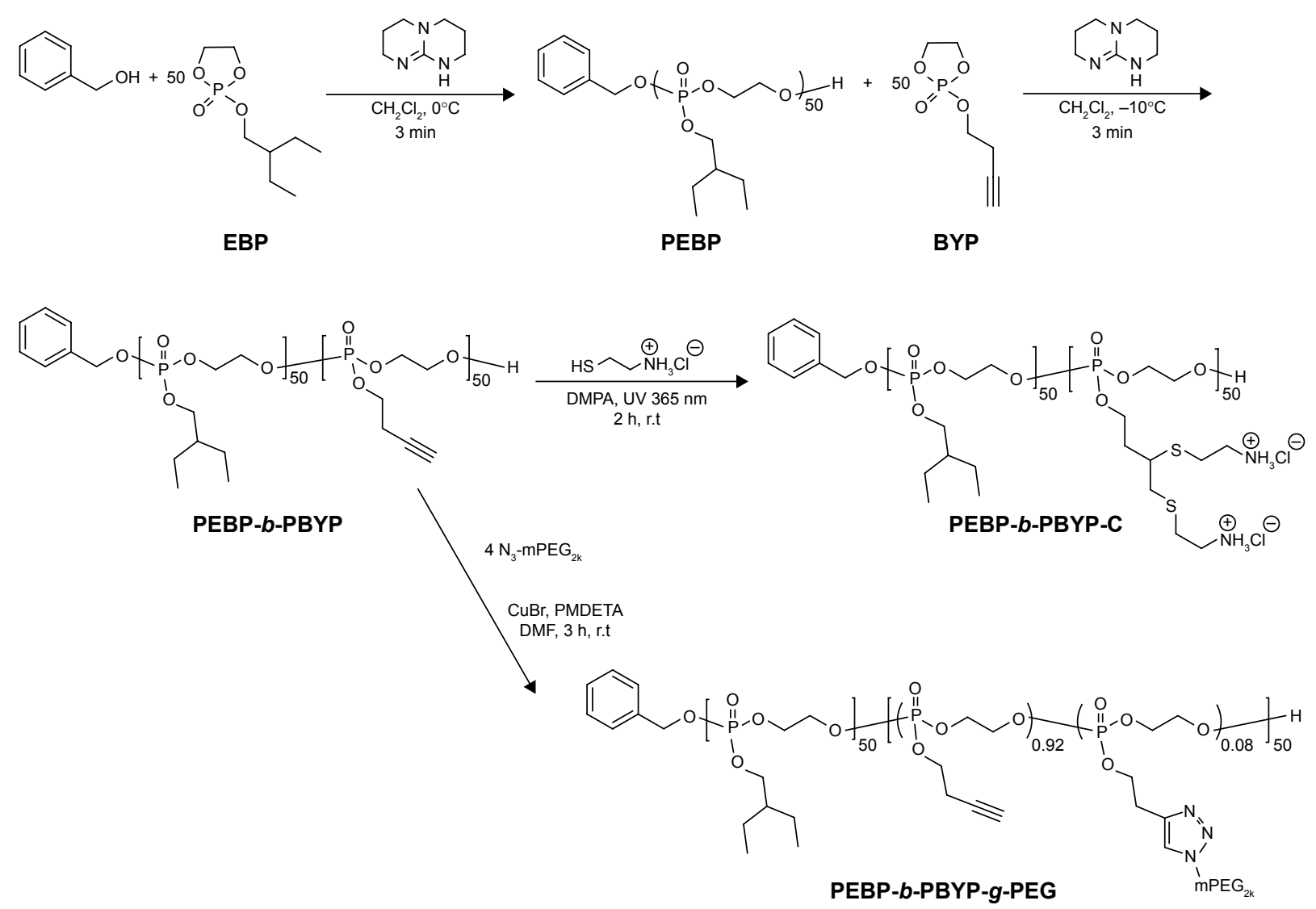

Scheme I Synthesis of the cationic block copolymer PEBP-b-PBYP-C via thiol-yne click reactions and the PEGylated terpolymer PEBP-b-PBYP-g-PEG via copper(I)-catalyzed alkyne-azide cycloaddition click reactions.

Abbreviations: PEBP-b-PBYP, poly(2-ethylbutoxy phospholane)-block-poly(2-butynyl phospholane); PEG, polyethylene glycol; EBP, ethylbutoxy phospholane; BYP, 2-butynyl

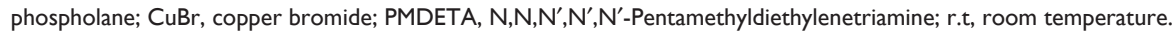

also be easily modified with cysteamine hydrochloride to afford the formation of cationic diblock copolymer via thiolyne click reactions (Scheme 1). Excess equivalents of thiols to alkyne groups were used in the DMPA photoinitiated radical reaction in order to completely transform the alkynes into amines. After $2 \mathrm{~h}$ of exposure to UV irradiation at $365 \mathrm{~nm}$, the reaction mixture was transferred to dialysis tubing (MWCO: $12-14 \mathrm{kDa}$ ) and dialyzed against nanopure water with hydrochloride $(\mathrm{pH} 3.0)$ in the cold room $\left(4^{\circ} \mathrm{C}-8^{\circ} \mathrm{C}\right)$ for $36 \mathrm{~h}$ to remove excess cysteamine hydrochloride and other small molecules. After purification, the purified cationic polymer was lyophilized and stored in the $-20^{\circ} \mathrm{C}$ freezer for further use as siRNA carrier. The ${ }^{1} \mathrm{H} N M R$ and ${ }^{31} \mathrm{P}$ NMR spectra of the cationic block copolymer PEBP- $b$-PBYP-C and PEGylated terpolymer PEBP- $b$-PBYP-g-PEG are inlcuded in Figures $\mathrm{S} 1$ and $\underline{\mathrm{S} 2}$.

The purpose of this study was to combine two types of PPE nanoparticles, for the combined delivery of chemotherapeutics and nucleic acids. For delivery of chemotherapeutics, PEBP- $b$-PBYP-g-PEG nanoparticles with hydrophobic cores and neutral surfaces were prepared. Sorafenib and paclitaxel were selected for the potential treatment of HCC, and several optimization steps were carried out to maximize the loading of the drugs into the nanoparticles. The optimization procedures involved the use of various organic solvents (acetonitrile, ethanol, etc), methods of evaporation (heat or vacuum) and loading the drugs separately or combined. It was found that using ethanol followed by vacuum evaporation resulted in monodispersed nanoparticles with efficient loading of sorafenib and/or paclitaxel. Cationic PPE nanoparticles were prepared for electrostatic complexation with anionic siRNA at various N/P ratios, and several characterizations were performed to select nanoparticles with the optimal physicochemical and biological characteristics and stability.

\section{Preparation and characterizations of paclitaxel- and sorafenib-loaded nanoparticles}

One of the main advantages of the developed PPE nanoparticles is the ease of synthesis, preparation and loading with various drugs. In contrast to the most commonly used 
Table I Size (intensity-averaged hydrodynamic diameter), size distribution (PDI) and zeta potential measurements of the neutral PPE nanoparticles that either empty or loaded with sorafenib (SOR-PPE), paclitaxel (PTX-PPE) or both drugs (PTX-SOR-PPE)

\begin{tabular}{lllll}
\hline Nanoparticle & $\begin{array}{l}\text { Particle } \\
\text { size }(\mathbf{n m})\end{array}$ & PDI & $\begin{array}{l}\text { Zeta potential } \\
(\mathbf{m V})\end{array}$ & EE (\%) \\
\hline Empty & $22 \pm 1$ & $0.22 \pm 0.00$ & $-35.1 \pm 0.8$ & - \\
SOR-PPE & $35 \pm 1$ & $0.30 \pm 0.01$ & $-19.9 \pm 0.6$ & 99.3 \\
PTX-PPE & $36 \pm 1$ & $0.46 \pm 0.03$ & $-26.1 \pm 4.1$ & 98.4 \\
PTX-SOR-PPE & $31 \pm 1$ & $0.39 \pm 0.02$ & $-21.7 \pm 2.1$ & 95.0 \\
\hline
\end{tabular}

Notes: Data are presented as mean \pm standard deviation $(n=3)$. Drug entrapment efficiency in the nanoparticles is also presented as the encapsulation efficiency (EE, \%). Abbreviations: EE, entrapment efficiency; PDI, polydispersity index; PPE, polyphosphoester; PTX-PPE, paclitaxel-loaded polyphosphoester; PTX-SOR-PPE, paclitaxel- and sorafenib-loaded polyphosphoester; SOR-PPE, sorafenib-loaded polyphosphoester.

methods ${ }^{41}$ for loading chemotherapeutics into nanoparticles, where several technical procedures are involved in these experiments, paclitaxel, sorafenib or a mixture of the two drugs could be simply loaded into the nanoparticles via mixing of the drug and polymer in ethanol followed by the evaporation of the organic solvent and resuspension of the formed film in water. Nanoparticles, either empty or loaded with paclitaxel, sorafenib or both drugs were monodispersed nanoparticles of small size and narrow size distribution (Table 1; Figure 1). However, a significant increase in size was observed upon incorporation of drugs into the nanoparticles. Size and morphology of the PPE nanoparticles have been studied previously, where monodispersed and spherical nanoparticles have been illustrated. ${ }^{21,22,25}$ In this study, when paclitaxel-loaded nanoparticles were imaged under the TEM, spherical particles (diameter of $34 \pm 3 \mathrm{~nm}$ ) with well-defined edges and contrast have been demonstrated (Figure S3). The size obtained from the TEM images is in agreement with the dynamic light scattering data. Nanoparticles had negative zeta potential values and exceptionally high loading efficiency (95\%-99\%; Table 1). These results represent ca. 5800- and 2500-fold increase in the aqueous solubilities of sorafenib and paclitaxel, respectively, in addition to providing a combinational therapy by incorporating both drugs into
A

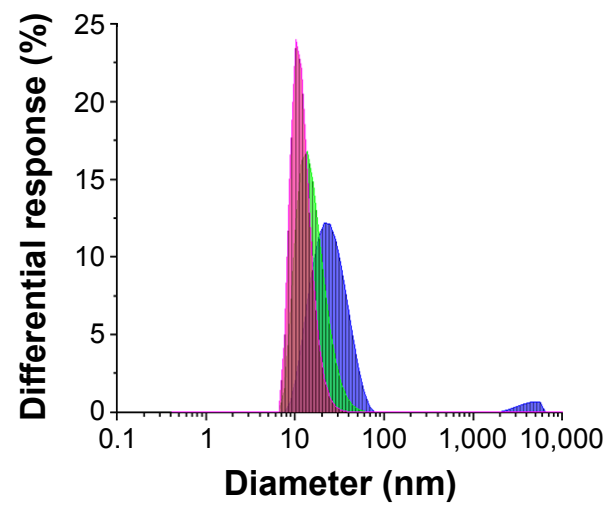

C

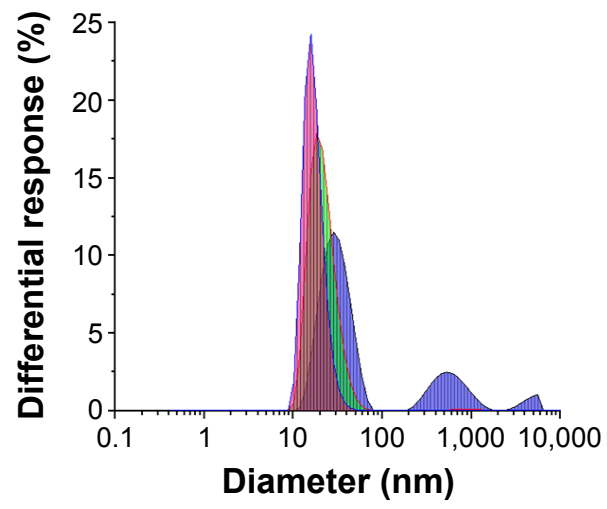

B

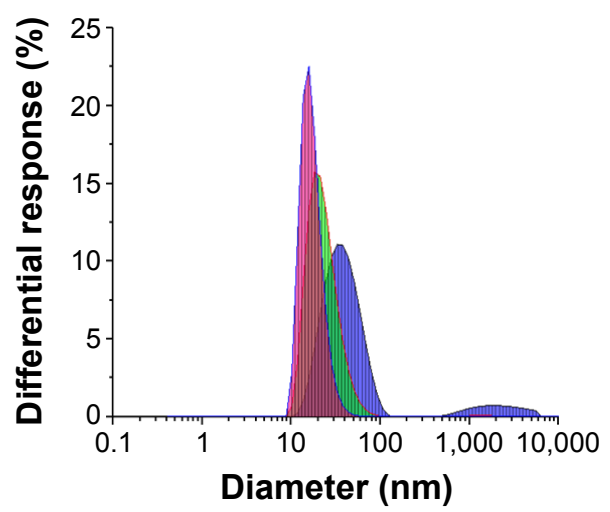

D

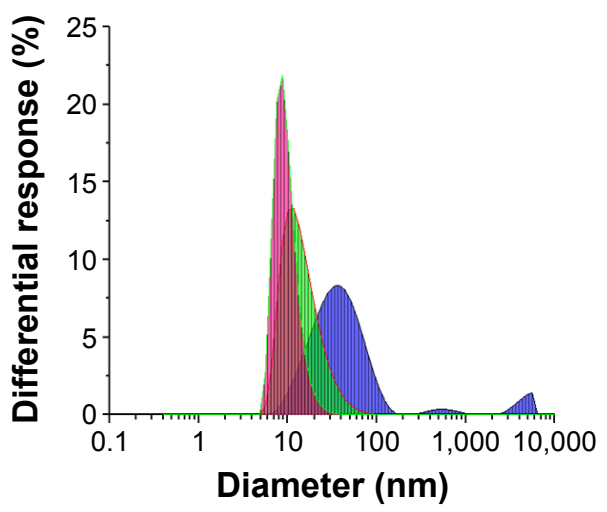

Differential intensity (\%) س Differential volume (\%) ए Differential number (\%)

Figure I Intensity-, volume- and number-averaged hydrodynamic diameter histograms of empty nanoparticles (A), SOR-PPE (B), PTX-PPE (C) and PTX-SOR-PPE (D) nanoparticles.

Abbreviations: PTX-SOR-PPE, paclitaxel- and sorafenib-loaded polyphosphoester; PTX-PPE, paclitaxel-loaded polyphosphoester; SOR-PPE, sorafenib-loaded polyphosphoester. 
N/P:

$\begin{array}{llllllllllllll}0 & 0.5 & 1 & 2 & 3 & 4 & 5 & 10 & 15 & 20 & 25 & 30 & 35 & 40\end{array}$

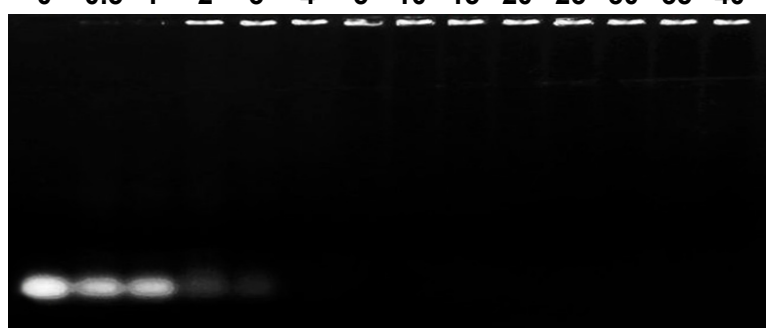

Figure $2 \mathrm{Gel}$ retardation assay of siRNA that either free (N/P ratio of 0 ) or complexed to cationic PPE polymer at different N/P ratios $(0.5-40)$ using I\% agarose gel. Note: Complete retardation of the siRNA migration was observed at N/P ratios of 4-40.

Abbreviations: N/P, nitrogen-to-phosphate; PPE, polyphosphoester; siRNA, short interfering RNA.

the same vehicle. We have previously utilized PPE-based polymeric nanostructures either for physical or chemical loading of paclitaxel and achieved ultrahigh loading of the drug within the nanostructures. ${ }^{25,34,42}$

\section{Preparation and charactericzation of siRNA-loaded nanoparticles}

Increasing amounts of the cationic PPE polymer were mixed with fixed amount of siRNA, and gel retardation assay was utilized to evaluate the complexation efficiency of the polymer at various N/P ratios. The siRNA bands showed complete migration at $\mathrm{N} / \mathrm{P}$ ratios of 0.5 and 1 , similar to the migration profile of the naked siRNA (N/P ratio of 0 ), due to the inefficient encapsulation at these low ratios. At N/P ratios of 2 and 3 , partial complexation of siRNA with the PPE polymer was observed. At N/P ratios ranging from 4 to 40 , siRNA migration was completely retarded due to the efficient complexation between the siRNA and the polymer (Figure 2). We have demonstrated previously that chitosan and PEI could efficiently complex siRNA at N/P ratios of 3 and 5, respectively. ${ }^{26}$ Hence, the cationic PPE polymer could efficiently complex siRNA at similar N/P ratios to the other commonly utilized cationic vectors (ie, chitosan and PEI).

Nanoparticles were prepared at N/P ratios ranging from 5 to 40 , with a constant volume of siRNA $(1.5 \mu \mathrm{L})$ and different amounts of PPE polymer. The sizes and zeta potential values of the nanoparticles were determined (Figure 3). The size of the nanoparticles prepared at different N/P ratios ranged from 170 to $400 \mathrm{~nm}$ (Figure 3A). Upon the addition of PPE, the size of nanoparticles initially increased to $390 \mathrm{~nm}$. At N/P ratio of 5, a slight decrease in size occurred to reach $280 \mathrm{~nm}$. Then, the reduction in size continued upon increasing the $\mathrm{N} / \mathrm{P}$ ratio. A similar trend was observed for the PDI values. As expected, the zeta potential values increased upon increasing the $\mathrm{N} / \mathrm{P}$ ratio (Figure 3B). Nanoparticles prepared at N/P ratio of $15 \mathrm{had}$ zeta potential of $30 \mathrm{mV}$. With further increase in the N/P ratio, a slight increase was observed for the zeta potential. PPE/siRNA nanoparticles prepared at N/P ratio of 25 had hydrodynamic diameter of ca. $180 \mathrm{~nm}$, PDI of 0.25 and zeta potential of ca. $+38 \mathrm{mV}$. These nanoparticles were selected for further studies due to their small size, low PDI and high zeta potential, which might improve nanocomplexes stability. Similar optimizations for the chitosan/Tpp and PEI complexes with siRNA were performed, as reported previously, and the optimal ratio was selected at N/P ratio of 45 for both types of nanoparticles. ${ }^{26}$ The zeta potential values for the chitosan/Tpp and PEI nanoparticles at N/P ratio of 45 were $22.7 \pm 1.6$ and $34.9 \pm 1.9 \mathrm{mV}$, respectively. Chitosan/Tpp and PEI nanoparticles prepared at N/P ratio of
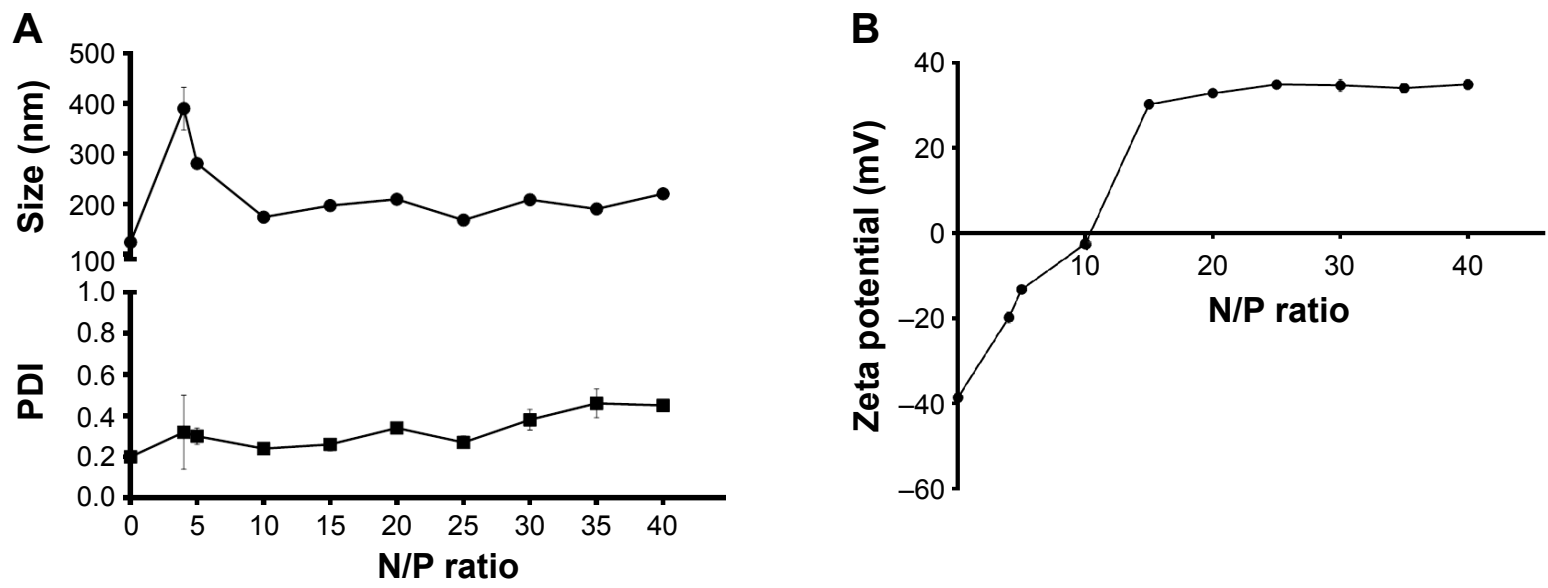

Figure 3 The effect of N/P ratio on the size and size distribution (PDI) (A) and zeta potential values (B) of siRNA-PPE nanoparticles. Note: The values are presented as mean \pm standard deviation $(n=3)$.

Abbreviations: N/P, nitrogen-to-phosphate; PDI, polydispersity index; PPE, polyphosphoester; siRNA, short interfering RNA. 
45 had hydrodynamic diameters of $340 \pm 10$ and $110 \pm 10 \mathrm{~nm}$, and PDI of $0.47 \pm 0.03$ and $0.37 \pm 0.02$, respectively.

Measurements of size and PDI in solutions of increasing concentrations of $\mathrm{NaCl}$ and dextrose were carried out to evaluate the stability of the nanoparticles. A significant increase in the size and PDI of nanoparticles prepared at N/P ratio of 25 was observed upon increasing the $\mathrm{NaCl}$ concentration (Figure 4A). The size increased from 170 to $220 \mathrm{~nm}$ at the physiological ionic strength, which is equivalent to $0.15 \mathrm{M}$ (Figure 4A). PDI also showed an increase from 0.25 to 0.8 . Slight changes in size and PDI of the nanoparticles prepared at N/P ratio of 25 were observed upon increasing the concentration of dextrose solutions to 5\% (Figure 4B). However, further increase in the concentrations of dextrose resulted in significant increase in the size. Given the fact that the physiological concentrations of saline and dextrose that are commonly utilized for intravenous injections are $0.15 \mathrm{M}$ for $\mathrm{NaCl}$ and $5 \%$ for dextrose; reconstitution of the nanoparticles in these solutions should not significantly affect their stability and physicochemical characteristics, in particular, in dextrose solutions. The difference in stability in $\mathrm{NaCl}$ and dextrose solutions might be because of the screening of charges on the cationic PPE nanoparticles by $\mathrm{NaCl}$ salt, and thus resulting in dissociation of the polyionic nanocomplexes. ${ }^{43}$ It might be also because of the hydrolytic degradation of the phosphoester backbone. ${ }^{44}$ siRNA-loaded chitoan/Tpp nanoparticles and PEI nanoparticles also had high stability in saline and dextrose solutions of different concentrations (data not shown).

\section{Effect of freeze-drying}

The effect of freeze-drying on stability of the nanoparticles was studied (Tables S1-S3). The size and size distribution (PDI) were measured before and after freeze-drying in the presence of several cryoprotectants at different quantities. The size of PTX-SOR-PPE nanoparticles significantly increased in the absence of cryoprotectants $(216 \mathrm{~nm})$ and in the presence of $2 \%$ mannitol $(626 \mathrm{~nm})$, as compared to the size of nanoparticles before freeze-drying $(30 \mathrm{~nm})$. However, a slight increase in size was observed in the presence of $0.1 \%$ of mannitol (46 nm). Regarding the siRNA-PPE nanoparticles, addition of mannitol $(1 \mathrm{mg} / \mathrm{mL})$ resulted in a reduction of the size from 155 to $125 \mathrm{~nm}$ and an increase in the PDI from 0.17 to 0.52 , probably because of a slight aggregation during the freeze-drying. Addition of sucrose (10\%) resulted in an increase in the size from 117 to $208 \mathrm{~nm}$. Interestingly, the nanoparticles could maintain their size and PDI without the addition of any cryoprotectants. Polyionic complexes formed between siRNA and PEI, poly(amidoamine)/poly(ethylene glycol)-b-poly(propyl methacrylate-co-methacrylic acid) or aminated poly(glycerol methacrylate) polymers also maintained their size characteristics upon freeze-drying without the addition of cryoprotectants, whereas complexes between siRNA and chitosan/Tpp polymers aggregated upon freezedrying, in the presence or absence of cryoprotectants. ${ }^{26,45}$

\section{Effect of physical mixing of the neutral and cationic PPE nanoparticles}

Neutral and cationic PPE nanoparticles could be loaded with anticancer drugs and siRNA, respectively, and could be freeze-dried, and their size characteristics were maintained after reconstitution in water and in physiological solutions. The two kinds of nanoparticles, paclitaxel-/sorafenib-loaded neutral PPE and siRNA-loaded cationic PPE nanoparticles, were mixed together, and size distributions of the two nanoparticles were measured (Figure 5). Interestingly, the two types of nanoparticles could maintain their sizes and size distributions after being mixed (Table S4). Although the
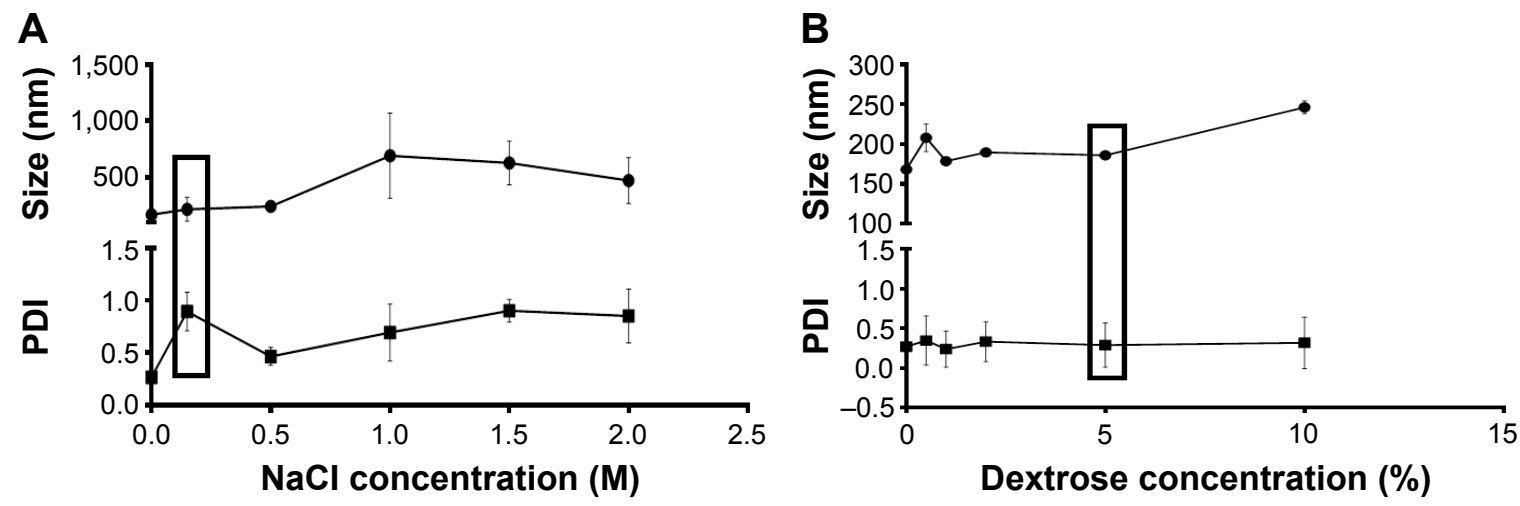

Figure 4 Effect of concentrations of $\mathrm{NaCl}(\mathbf{A})$ and dextrose $(\mathbf{B})$ solutions on the size and PDI of siRNA-PPE nanoparticles prepared at N/P ratio of 25 .

Notes: The values are presented as mean \pm standard deviation $(n=3)$. The insets in the figures (boxes) indicate the physiological concentrations of $\mathrm{NaCl}$ and dextrose solutions.

Abbreviations: N/P, nitrogen-to-phosphate; PDI, polydispersity index; PPE, polyphosphoester; siRNA, short interfering RNA. 

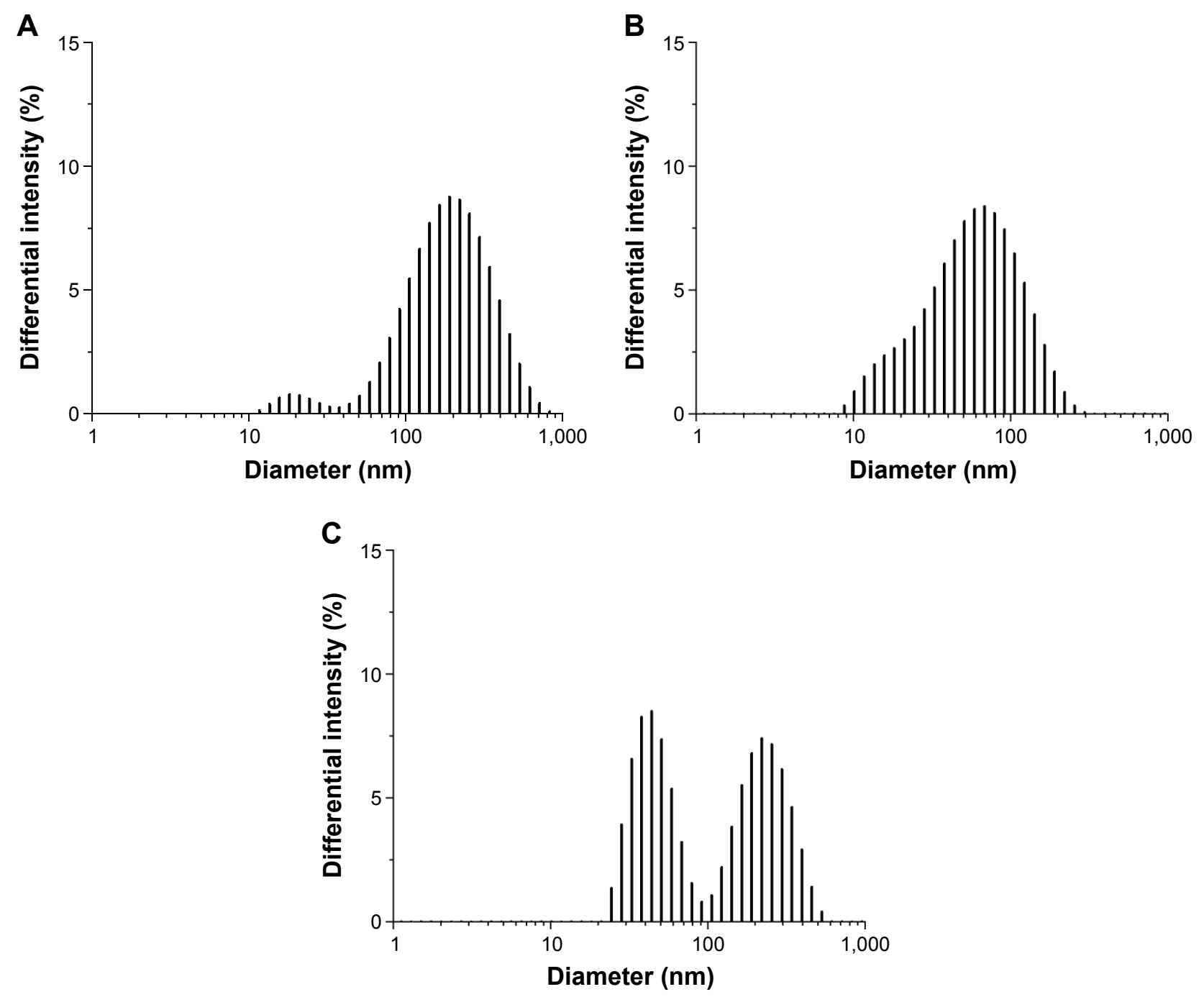

Figure 5 Intensity-averaged hydrodynamic diameter histograms of siRNA-loaded PPE nanoparticles (A), PTX-SOR-PPE nanoparticles (B), and mixture of the two types of nanoparticles (C).

Abbreviations: PPE, polyphosphoester; PTX-SOR-PPE, paclitaxel- and sorafenib-loaded polyphosphoester; siRNA, short interfering RNA.

cationic nanoparticles might be able to complex siRNA and contain the anticancer drugs in their hydrophobic cores, it is better to load drugs and siRNA separately into two nanoparticles. In this way, mixing of the two drugs and siRNA at different ratios can be precisely controlled according to therapeutic needs. In addition, nucleic acids are sensitive to the harsh conditions (organic solvent and evaporation) used for preparing the nanoparticles loaded with hydrophobic anticancer drugs. This could be a convenient way of administration of multiple drugs using PPE nanoparticles of different surface chemistries in clinical settings. The size of nanoparticles measured in this study is in agreement with the previously reported data by our group for the neutral PPE nanoparticles. ${ }^{21,22,25}$ However, nanocomplexes formed between siRNA and the cationic fully degradable PPE polymers have not been reported previously.

\section{Toxicity of the nanoparticles}

Intravenous administration of anticancer drugs is known to induce hemolysis of RBCs, and thus leading to anemia. ${ }^{46}$ Encapsulation of paclitaxel and sorafenib into the nanoparticles might prevent them from inducing hemolysis, whereas, in Taxol, paclitaxel is dissolved in the low molecular weight surfactant Cremophor EL and, thus, the drug is available to interact with blood cells. ${ }^{47}$ The effect of the drug-loaded nanoparticles on blood cells was studied by incubating a blood suspension with sorafenib- and paclitaxel-loaded PPE nanoparticles $(0.5 \mathrm{mg} / \mathrm{mL}$ paclitaxel and $0.5 \mathrm{mg} / \mathrm{mL}$ sorafenib) versus the Taxol mimicking formulation $(6 \mathrm{mg} / \mathrm{mL}$ paclitaxel). The nanoparticles and saline did not induce hemolysis, whereas the Taxol mimicking formulation resulted in significant hemolysis to blood cells as indicated by the released hemoglobin from the ruptured cells (Figure S4). 
Taxol was also prepared as the market mimicking formulation according to the manufacturer instructions but with slight modifications to contain $0.5 \mathrm{mg} / \mathrm{mL}$ paclitaxel and $0.5 \mathrm{mg} / \mathrm{mL}$ sorafenib (ie, same concentrations in the tested nanoparticles). Even at this diluted concentration of Taxol, slight hemolysis was observed when it was incubated with RBCs (Figure S5). Cytotoxicities of the PPE nanoparticles loaded with either paclitaxel or sorafenib or both drugs were evaluated in huh-7 cells. The $\mathrm{IC}_{50}$ values for the paclitaxeland sorafenib-loaded PPE nanoparticles in huh-7 cells were 1.9 and $3.4 \mu \mathrm{M}$, respectively. The determined $\mathrm{IC}_{50}$ values are in agreement with values reported in the literature. ${ }^{34,48-50}$ When both drugs were combined into the same PPE nanoparticles, slightly higher $\mathrm{IC}_{50}$ values were observed (ie, 2.9 and $5.4 \mu \mathrm{M}$ for paclitaxel and sorafenib, respectively). Overall, these data demonstrate the initial ability of nanoparticles to release the embedded cargoes and induce significant cytotoxicities in the tested cell line. It has been demonstrated before that these nanoparticles can release the anticancer drug paclitaxel in a slow and sustained release manner. ${ }^{25}$ Worth mentioning is that the empty PPE nanoparticles did not result in cytotoxicity at the range of the tested concentrations. The next step was to evaluate the in vivo toxicity of PPE nanoparticles. Cationic PPE polymer was selected because cationic polymers are known to induce significant toxicities both in vitro and in vivo, as compared to nanoparticles with neutral or anionic surface charges. ${ }^{22,51}$ In vivo toxicity of the cationic PPE, chitosan/Tpp and PEI polymers was assessed after intraperitoneal injection of increasing concentrations of the polymers into mice. The animals were observed for $24 \mathrm{~h}$, the number of deceased mice was counted (Figure 6), and the $\mathrm{LD}_{50}$ was calculated using the graphical method of Miller and Tainter. ${ }^{27-29} \mathrm{PEI}$ toxicity increased with increasing

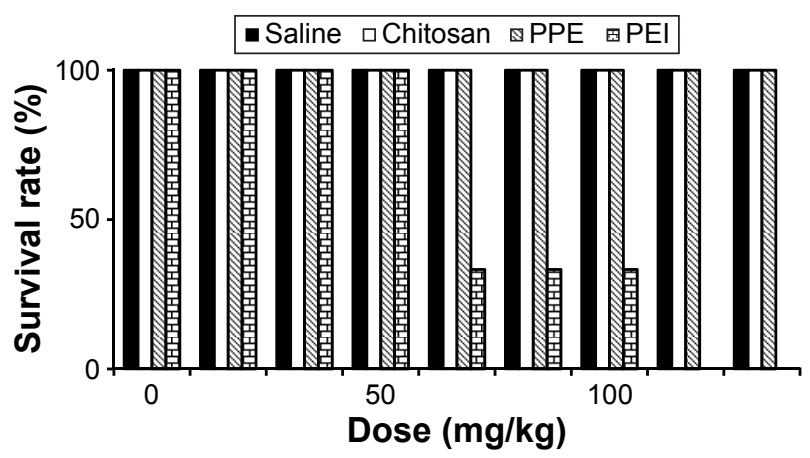

Figure 6 Survival rates of mice treated with escalating doses of PPE, chitosan/TPP and PEI nanoparticles after intraperitoneal injection.

Notes: The endpoint of the experiment was $24 \mathrm{~h}$. In vivo distribution of the siRNAloaded PPE nanoparticles.

Abbreviations: PEI, polyethylenimine; PPE, polyphosphoester; siRNA, short interfering RNA; Tpp, tripolyphosphate. the concentration of the injected polymer in mice (calculated $\mathrm{LD}_{50}$ of $\left.61.7 \mathrm{mg} / \mathrm{kg}\right) .{ }^{26}$ On the contrary, PPE and chitosan polymers did not induce toxicity and no animals died at the highest tested concentration (ie, $133.3 \mathrm{mg} / \mathrm{kg}$ ). Degradability has a great impact on the safety profiles of nanoparticles, and degradable nanoparticles are usually associated with lower toxicity both in vitro and in vivo. ${ }^{22,26,52}$

Cationic PPE, chitosan/Tpp and PEI polymers were complexed with cyanine 5-fluorescently labeled siRNA to form the nanoparticles before intravenous injection of the siRNA, that is, either naked or complexed to the polymers in mice at a dose of $0.5 \mathrm{mg}$ siRNA/ $\mathrm{kg}$. The mice were sacrificed after $2 \mathrm{~h}$ of the injection and specimens from the liver, kidney, spleen, lungs and heart of control and treated mice were harvested and processed for imaging under fluorescence and light microscopy to evaluate the in vivo delivery of siRNA and for histologic examination of the main organs for toxicity, respectively, as reported previously. ${ }^{26}$ Examination of hematoxylin and eosin-stained sections of the organs of control mice receiving naked siRNA has demonstrated normal hepatic, renal, cardiac, pulmonary and splenic architectures (data not shown). The histologic structures of the liver, cardiac muscles and spleen of mice treated with siRNA-loaded PPE nanoparticles appeared similar to the control groups (Figure 7). In the kidney, the proximal convoluted tubules showed sign of degeneration in the form of deeply stained nuclei and destructed brush border. Regarding the lung alveoli, the internal alveolar septum appeared congested with blood. These positive attributes demonstrated that these nanoconjugates can be used as a promising platform for drug delivery after further in vivo biological evaluations. Treatment with siRNA-loaded chitosan/Tpp and PEI nanoparticles led to obvious histologic changes, as reported previously by our group. ${ }^{26}$

No fluorescence was detected in the organs of the mice injected with the naked siRNA (Figure 8A). Rapid degradation of nucleic acids either in vitro upon incubation with serum or nucleases or in vivo after intravenous administration has been reported previously. ${ }^{45,52,53}$ DNA/RNA are vulnerable to intra- and extracellular nucleases, which degrade them rapidly and prevent them from reaching their target sites. Examination of unstained paraffin sections treated with siRNA-loaded PPE nanoparticles of liver, heart, kidney, lungs and spleen using fluorescence microscopy showed red fluorescence of varying intensities in the different organs (Figure 8B). Injection of the siRNA complexed with PEI resulted in strong uptake in the main organs. ${ }^{26}$ However, no selective uptake was observed. In addition, the high 


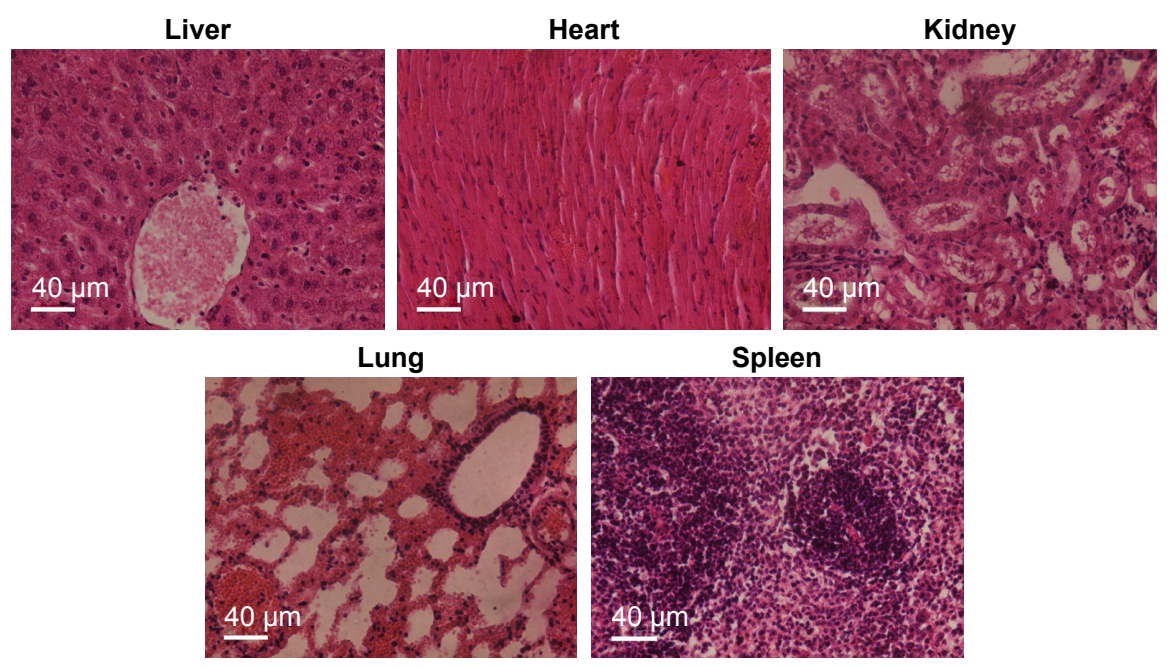

Figure 7 Photomicrographs of the histologic structures of liver, heart, kidney, lung and spleen of mice treated with siRNA-loaded PPE nanoparticles. Abbreviations: PPE, polyphosphoester; siRNA, short interfering RNA.

A

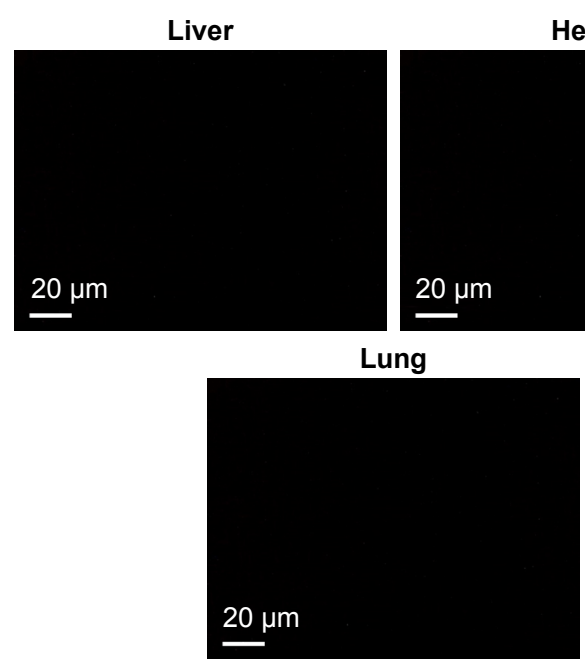

B
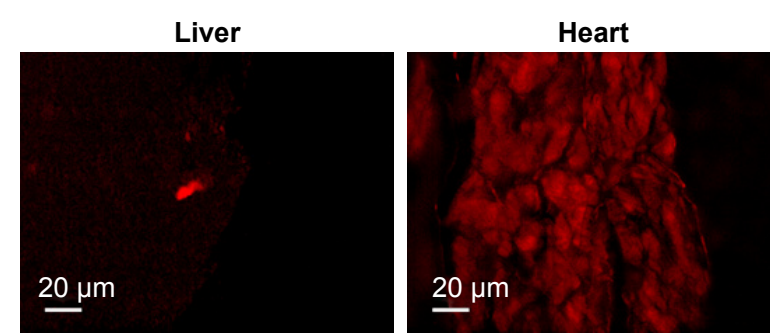

Lung
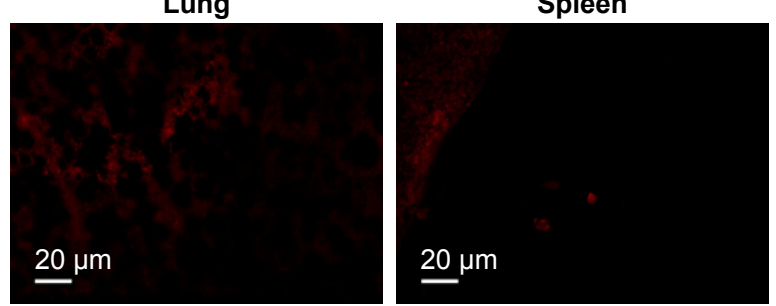

Kidney

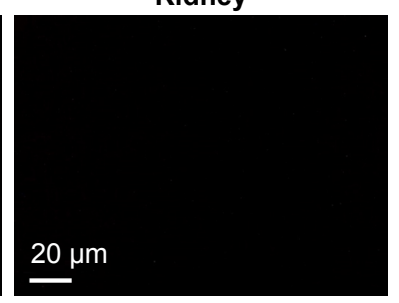

Spleen
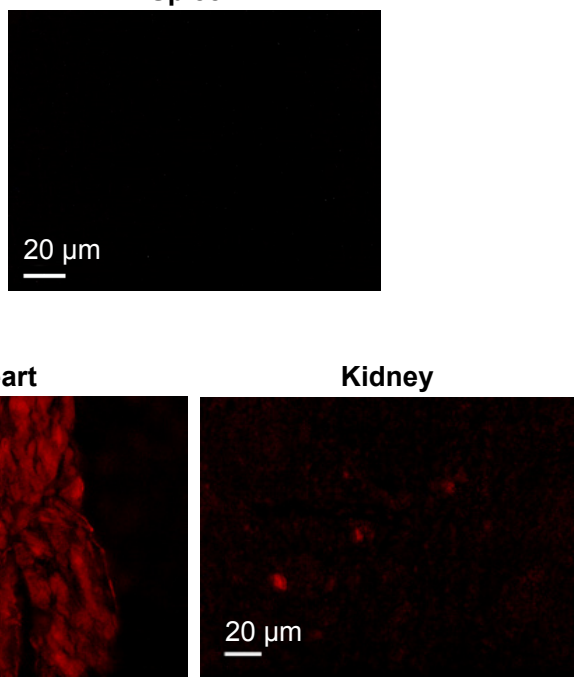

Spleen

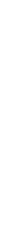

Figure 8 Biodistribution of cyanine 5-labeled siRNA that is either free (A) or loaded into PPE nanoparticles (B) in mice after intravenous injection of a dose of 0.5 mg siRNA/kg.

Abbreviations: PPE, polyphosphoester; siRNA, short interfering RNA. 
accumulation in the organs is compromised by the high toxicity in the main organs and low $\mathrm{LD}_{50}$ value of PEI. Chitosan-/ Tpp-based nanoparticles resulted in lower uptake in the main organs, as compared to the siRNA-PPE nanoparticles, which might be because of the relatively larger size of the chitosan nanoparticles as compared to the PPE- and PEI-based nanoparticles. ${ }^{26}$ Although the advantages of chitosan reported in our study, in terms of degradability and biocompatibility, it might not be able to deliver therapeutic concentrations of the loaded siRNA cargo to the target organs. We have selected the siRNA for the preliminary in vivo study because it is more vulnerable to nucleases and usually degrades within a few minutes after administration. In addition, it was easier to fluorescently label the siRNA and to visualize the siRNA biodistribution in vivo. Herein, we confirmed that siRNA could partially maintain stability in the blood, when protected in the nanoparticles, and be delivered to the various organs, as compared to the naked siRNA.

\section{Conclusion}

Cationic PPE polymers could efficiently complex siRNA, and stability of the nanoparticles could be maintained in physiological solutions and upon freeze-drying. When injected intravenously, the cationic nanoparticles were able to deliver siRNA into the main organs of mice. Neutral PPE nanoparticles were able to encapsulate two hydrophobic drugs, namely, sorafenib and paclitaxel, which are commonly used for the treatment of HCC. Mixing the neutral and cationic PPE nanoparticles did not result in precipitation, and the size characteristics of both types of nanoparticles were maintained. Hence, PPE might provide promising vehicles for the delivery of multiple drugs to diseased tissues via simple synthesis of the individual polymers and assembly into nanoparticles that can host several drugs in the same or different PPE nanoparticles. Avoiding sophisticated procedures in synthesis, drug-loading and nanoparticle assembly and mixing is of great importance for industrial and clinical implications. In vivo therapeutic efficiency of the combined formulations, loaded with paclitaxel, sorafenib and VEGF or integrin-targeted siRNA, will be pursued for the management of $\mathrm{HCC}$.

\section{Acknowledgments}

We gratefully acknowledge financial support from the Egyptian Science and Technology Development Fund (STDF, Demand-driven Project 5688 and Reintegration Grant 5362). Financial support from the Robert A. Welch Foundation (Grant No A-0001) is gratefully acknowledged.

\section{Disclosure}

The authors report no conflicts of interest in this work.

\section{References}

1. Elsabahy M, Heo GS, Lim SM, Sun G, Wooley KL. Polymeric nanostructures for imaging and therapy. Chem Rev. 2015;115(19): 10967-11011.

2. Elsabahy M, Wooley KL. Cytokines as biomarkers of nanoparticle immunotoxicity. Chem Soc Rev. 2013;42(12):5552-5576.

3. Elsabahy M, Wooley KL. Design of polymeric nanoparticles for biomedical delivery applications. Chem Soc Rev. 2012;41(7):2545-2561.

4. Chen Y, Wu JJ, Huang L. Nanoparticles targeted with NGR motif deliver c-myc siRNA and doxorubicin for anticancer therapy. Mol Ther. 2010;18(4):828-834.

5. Xiong XB, Lavasanifar A. Traceable multifunctional micellar nanocarriers for cancer-targeted co-delivery of MDR-1 siRNA and doxorubicin. ACS Nano. 2011;5(6):5202-5213.

6. Reddy TL, Garikapati KR, Reddy SG, et al. Simultaneous delivery of Paclitaxel and Bcl-2 siRNA via pH-Sensitive liposomal nanocarrier for the synergistic treatment of melanoma. Sci Rep. 2016;6:35223.

7. Sun TM, Du JZ, Yao YD, et al. Simultaneous delivery of siRNA and paclitaxel via a "two-in-one" micelleplex promotes synergistic tumor suppression. ACS Nano. 2011;5(2):1483-1494.

8. Chen AM, Zhang M, Wei D, et al. Co-delivery of doxorubicin and Bcl-2 siRNA by mesoporous silica nanoparticles enhances the efficacy of chemotherapy in multidrug-resistant cancer cells. Small. 2009; 5(23):2673-2677.

9. Elsabahy M, Shrestha R, Clark C, Taylor S, Leonard J, Wooley KL. Multifunctional hierarchically assembled nanostructures as complex stage-wise dual-delivery systems for coincidental yet differential trafficking of siRNA and paclitaxel. Nano Lett. 2013;13(5): 2172-2181.

10. Kloover JS, den Bakker MA, Gelderblom H, van Meerbeeck JP. Fatal outcome of a hypersensitivity reaction to paclitaxel: a critical review of premedication regimens. Br J Cancer. 2004;90(2):304-305.

11. Al-Rajabi R, Patel S, Ketchum NS, et al. Comparative dosing and efficacy of sorafenib in hepatocellular cancer patients with varying liver dysfunction. J Gastrointest Oncol. 2015;6(3):259-267.

12. Fitzmorris P, Shoreibah M, Anand BS, Singal AK. Management of hepatocellular carcinoma. J Cancer Res Clin Oncol. 2015;141(5): 861-876.

13. Kuo TC, Lu HP, Chao CC. The tyrosine kinase inhibitor sorafenib sensitizes hepatocellular carcinoma cells to taxol by suppressing the HURP protein. Biochem Pharmacol. 2011;82(2):184-194.

14. Dal Lago L, D'Hondt V, Awada A. Selected combination therapy with sorafenib: a review of clinical data and perspectives in advanced solid tumors. Oncologist. 2008;13(8):845-858.

15. Liu C, Chen Z, Chen Y, et al. Improving oral bioavailability of sorafenib by optimizing the "Spring" and "Parachute" based on molecular interaction mechanisms. Mol Pharm. 2016;13(2):599-608.

16. Wang XQ, Fan JM, Liu YO, Zhao B, Jia ZR, Zhang Q. Bioavailability and pharmacokinetics of sorafenib suspension, nanoparticles and nanomatrix for oral administration to rat. Int J Pharm. 2011; 419(1-2):339-346.

17. Raskopf E, Vogt A, Sauerbruch T, Schmitz V. siRNA targeting VEGF inhibits hepatocellular carcinoma growth and tumor angiogenesis in vivo. J Hepatol. 2008;49(6):977-984.

18. Bogorad RL, Yin H, Zeigerer A, et al. Nanoparticle-formulated siRNA targeting integrins inhibits hepatocellular carcinoma progression in mice. Nat Commun. 2014;5:3869.

19. Elsabahy M, Nazarali A, Foldvari M. Non-viral nucleic acid delivery: key challenges and future directions. Curr Drug Deliv. 2011;8(3): 235-244.

20. Mohamed NK, Hamad MA, Hafez MZ, Wooley KL, Elsabahy M. Nanomedicine in management of hepatocellular carcinoma: challenges and opportunities. Int J Cancer. Epub 2016 Nov 15. 
21. Zhang S, Zou J, Zhang F, et al. Rapid and versatile construction of diverse and functional nanostructures derived from a polyphosphoester-based biomimetic block copolymer system. J Am Chem Soc. 2012;134(44): 18467-18474.

22. Elsabahy M, Zhang S, Zhang F, et al. Surface charges and shell crosslinks each play significant roles in mediating degradation, biofouling, cytotoxicity and immunotoxicity for polyphosphoester-based nanoparticles. Sci Rep. 2013;3:3313.

23. Mao CQ, Du JZ, Sun TM, et al. A biodegradable amphiphilic and cationic triblock copolymer for the delivery of siRNA targeting the acid ceramidase gene for cancer therapy. Biomaterials. 2011;32(11):3124-3133.

24. Wang HX, Xiong MH, Wang YC, Zhu J, Wang J. N-acetylgalactosamine functionalized mixed micellar nanoparticles for targeted delivery of siRNA to liver. J Control Release. 2013;166(2):106-114.

25. Zhang F, Zhang S, Pollack SF, et al. Improving paclitaxel delivery: in vitro and in vivo characterization of PEGylated polyphosphoesterbased nanocarriers. J Am Chem Soc. 2015;137(5):2056-2066.

26. Morsi NG, Ali SM, Elsonbaty SS, et al. Poly(glycerol methacrylate)based degradable nanoparticles for delivery of small interfering RNA. Pharm Dev Technol. In press 2017.

27. DePass LR. Alternative approaches in median lethality (LD50) and acute toxicity testing. Toxicol lett. 1989;49(2-3):159-170.

28. Van den Heuvel MJ, Clark DG, Fielder RJ, et al. The international validation of a fixed-dose procedure as an alternative to the classical LD50 test. Food Chem Toxicol. 1990;28(7):469-482.

29. Miller LC, Tainter M. Estimation of the ED50 and its error by means of logarithmic-probit graph paper. Exp Biol Med. 1944;57(2):261-264.

30. Haroon MM, Dar GH, Jeyalakshmi D, et al. A designed recombinant fusion protein for targeted delivery of siRNA to the mouse brain. J Control Release. 2016;228:120-131.

31. Sato Y, Note Y, Maeki M, et al. Elucidation of the physicochemical properties and potency of siRNA-loaded small-sized lipid nanoparticles for siRNA delivery. J Control Release. 2016;229:48-57.

32. Zhang F, Smolen JA, Zhang S, et al. Degradable polyphosphoesterbased silver-loaded nanoparticles as therapeutics for bacterial lung infections. Nanoscale. 2015;7(6):2265-2270.

33. Liu JY, Huang W, Pang Y, Zhu X, Zhou Y, Yan D. The in vitro biocompatibility of self-assembled hyperbranched copolyphosphate nanocarriers. Biomaterials. 2010;31(21):5643-5651.

34. Zou J, Zhang F, Zhang S, et al. Poly(ethylene oxide)-blockpolyphosphoester-graft-paclitaxel conjugates with acid-labile linkages as a $\mathrm{pH}$-sensitive and functional nanoscopic platform for paclitaxel delivery. Adv Healthc Mater. 2014;3(3):441-448.

35. Li A, Luehmann HP, Sun G, et al. Synthesis and in vivo pharmacokinetic evaluation of degradable shell cross-linked polymer nanoparticles with poly(carboxybetaine) versus poly(ethylene glycol) surface-grafted coatings. ACS Nano. 2012;6(10):8970-8982.

36. Chen CK, Jones CH, Mistriotis P, et al. Poly(ethylene glycol)-blockcationic polylactide nanocomplexes of differing charge density for gene delivery. Biomaterials. 2013;34(37):9688-9699.

37. Quadir MA, Morton SW, Deng ZJ, et al. PEG-polypeptide block copolymers as $\mathrm{pH}$-responsive endosome-solubilizing drug nanocarriers. Mol Pharm. 2014;11(7):2420-2430.
38. He X, Fan J, Zhang F, et al. Multi-responsive hydrogels derived from the self-assembly of tethered allyl-functionalized racemic oligopeptides. J Mater Chem B Mater Biol Med. 2014;2(46):8123-8130.

39. Sun CY, Dou S, Du JZ, Yang XZ, Li YP, Wang J. Doxorubicin conjugate of poly(ethylene glycol)-block-polyphosphoester for cancer therapy. Adv Healthc Mater. 2014;3(2):261-272.

40. Sun TM, Du JZ, Yan LF, Mao HQ, Wang J. Self-assembled biodegradable micellar nanoparticles of amphiphilic and cationic block copolymer for siRNA delivery. Biomaterials. 2008;29(32):4348-4355.

41. Elsabahy M, Dufresne MH, Leroux JC. Polymeric micelles as versatile carriers for drugs and nucleic acids delivery. In: Torchilin V, Amiji M, editors. Materials for Nanomedicine. Hackensack, NY: Pan Stanford Publishing; 2010:169-234.

42. Zhang S, Zou J, Elsabahy M, et al. Poly(ethylene oxide)-blockpolyphosphester-based paclitaxel conjugates as a platform for ultrahigh paclitaxel-loaded multifunctional nanoparticles. Chem Sci. 2013;4(5):2122-2126.

43. Elsabahy M, Zhang M, Gan SM, Waldron KC, Leroux JC. Synthesis and enzymatic stability of PEGylated oligonucleotide duplexes and their self-assemblies with polyamidoamine dendrimers. Soft Matter. 2008; 4(2):294-302.

44. Wen J, Mao HQ, Li W, Lin KY, Leong KW. Biodegradable polyphosphoester micelles for gene delivery. J Pharm Sci. 2004;93(8): 2142-2157.

45. Elsabahy M, Wazen N, Puxan N, et al. Delivery of nucleic acids through the controlled disassembly of multifunctional nanocomplexes. $A d v$ Funct Mater. 2009;19(24):3862-3867.

46. Groopman JE, Itri LM. Chemotherapy-induced anemia in adults: incidence and treatment. J Natl Cancer Inst. 1999;91(19):1616-1634.

47. Lu J, Chuan X, Zhang H, et al. Free paclitaxel loaded PEGylated-paclitaxel nanoparticles: preparation and comparison with other paclitaxel systems in vitro and in vivo. Int J Pharm. 2014;471(1-2):525-535.

48. Kim DH, Kim MD, Choi CW, et al. Antitumor activity of sorafenibincorporated nanoparticles of dextran/poly(dl-lactide-co-glycolide) block copolymer. Nanoscale Res Lett. 2012;7(1):91.

49. Morisaki T, Umebayashi M, Kiyota A, et al. Combining celecoxib with sorafenib synergistically inhibits hepatocellular carcinoma cells in vitro. Anticancer Res. 2013;33(4):1387-1395.

50. Chen W, Wu J, Shi H, et al. Hepatic stellate cell coculture enables sorafenib resistance in Huh7 cells through HGF/c-Met/Akt and Jak2/ Stat3 pathways. Biomed Res Int. 2014;2014:764981.

51. Elsabahy M, Wooley KL. Data mining as a guide for the construction of cross-linked nanoparticles with low immunotoxicity via control of polymer chemistry and supramolecular assembly. Acc Chem Res. 2015; 48(6): 1620-1630.

52. Elsabahy M, Samarajeewa S, Raymond JE, Clark C, Wooley KL. Shellcrosslinked knedel-like nanoparticles induce lower immunotoxicity than their non-crosslinked analogs. J Mater Chem B Mater Biol Med. 2013;1(39):5241-5255.

53. Harada-Shiba M, Yamauchi K, Harada A, Takamisawa I, Shimokado K, Kataoka K. Polyion complex micelles as vectors in gene therapypharmacokinetics and in vivo gene transfer. Gene Ther. 2002;9(6): 407-414.
Drug Design, Development and Therapy

\section{Publish your work in this journal}

Drug Design, Development and Therapy is an international, peerreviewed open-access journal that spans the spectrum of drug design and development through to clinical applications. Clinical outcomes, patient safety, and programs for the development and effective, safe, and sustained use of medicines are the features of the journal, which

\section{Dovepress}

has also been accepted for indexing on PubMed Central. The manuscript management system is completely online and includes a very quick and fair peer-review system, which is all easy to use. Visit http://www.dovepress.com/testimonials.php to read real quotes from published authors. 